文章编号: 1001-3555(2020) 05-0407-08

\title{
砷中毒商业 $\mathrm{V}_{2} \mathrm{O}_{5}-\mathrm{WO}_{3} / \mathrm{TiO}_{2}$ 催化剂再生研究
}

\author{
赵重阳', 李国波 ${ }^{2}$, 眭华军 1 , 刘初明 1 , 王 玲 ${ }^{2}$, 张亚平 $2 *$ \\ (1. 国家能源集团棟壁发电厂, 江苏镇江. 212006; \\ 2. 东南大学 能源与环境学:院, 汇:苏南京 210096)
}

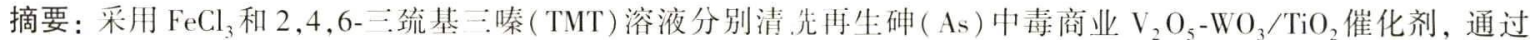
BET、XRD、XRF、 in situ DRIFTS 以及 $\mathrm{H}_{2}$-TPR 等表征方法对清洗再生前后催化剂理化性质进行分析. 研究发现, 清洗后催化剂脱硝活性有极大地恢复, $20 \mathrm{mg} \cdot \mathrm{mL}^{-1} \mathrm{FeCl}_{3}$ 和 $0.5 \%$ TMT 溶液再生 $30 \mathrm{~min}$ 时最佳 As 去除率分别为 83.67\% 和 94.57\% . 清洗后, 阻塞在催化剂微孔和中孔中的 AsO 被清除, 因此再生后催化剂比表面积和孔体积均 有所增大而平均孔径略有减小. 同时, $\mathrm{FeCl}_{3}$ 和 2,4,6-TMT 溶液清洗再生后催化剂表面 Brønsted 和 Lewis 酸强度均 有所增加, 这可能是再生催化剂催化性能提高的主要原因
\end{abstract}

关键词: SCR; 催化剂; As 中毒; 再生

中图分类号: 0643.32

文献标志码: A

近年来, $\mathrm{NH}_{3}$ 法选择性催化还原 $\left(\mathrm{NH}_{3}-\mathrm{SCR}\right)$ 已被 证明是控制燃煤 $\mathrm{NO}_{x}$ 排放的最有效的技术之一 ${ }^{11}$, $\mathrm{V}_{2} \mathrm{O}_{5}-\mathrm{WO}_{3} / \mathrm{TiO}_{2}$ 蜂窝型商用催化剂是 SCR 脱硝系统 使用最广泛的丁.业催化剂 ${ }^{2-3]}$. 众所周知, 催化剂是 SCR 脱硝系统的关键部分, 对整个系统 $\mathrm{NO}_{x}$ 的脱除 效率起着决定性作用. 通人 SCR 系统中的 $\mathrm{NH}_{3}$ 在催 化剂表面活性位点上吸附并活化, 将 $\mathrm{NO}_{x}$ 还原为 $\mathrm{N}_{2}$ 和 $\mathrm{H}_{2} \mathrm{O}$. 催化失活通常由被活性位点占据并覆盖导 致. 其中, As 是引起催化剂中毒失活的重要致毒元 素之一 ${ }^{[4}$. 目前, 在实验室层面已有针对 $\mathrm{V}_{2} \mathrm{O}_{5} / \mathrm{TiO}_{2}$ SCR 催化剂的 As 失活机理方面的许多研究 ${ }^{[5-6]}$.

在大多数煤种中, As 通常以硫化物 $\left(\mathrm{FeS}_{2} \mathrm{As}\right.$, $\left.\mathrm{As}_{2} \mathrm{~S}_{3}\right)$ 形式存在 ${ }^{[7-8]}$, 如次烟煤和褐煤中的 $A s$ 在燃 烧后转化为 $\mathrm{As}_{2} \mathrm{O}_{3}$ 挥发到空气中. $\mathrm{As}$ 中毒是脱硝催 化剂失活的主要原因, 因此许多学者对之展开研 究 ${ }^{9-12]}$. Peng 等 ${ }^{[9]}$ 采用实验与 DFT 计算结合的方 法, 研究发现催化剂 $1.4 \%$ As 中毒后导致 $\mathrm{N}_{2}$ 选择性 降低. 同时, $\mathrm{H}_{2} \mathrm{O}$ 存在的情况下, $\mathrm{NO}_{2}$ 转化率降低效

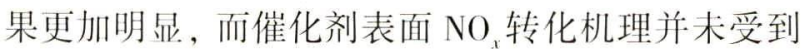
影响. Kong 等 ${ }^{[0]}$ 和 Jian 等 ${ }^{[11}$ 研究发现, $\mathrm{As}_{2} \mathrm{O}_{3}$ 会被
吸附在催化剂表面，部分被表面吸附氧氧化，并最 终转化为 $\mathrm{As}_{2} \mathrm{O}_{5}$. 值得注意的是, 整个氧化过程并 无 W 和 $\mathrm{Ti}$ 元素参与. 此外, 形成的 $\mathrm{As}_{2} \mathrm{O}_{5}$ 层沉积在 催化剂表面并阻碍催化剂对 $\mathrm{NH}_{3}$ 的吸附与活化. Sun 等 ${ }^{[2]}$ 从化学反应动力学的角度解释了 As 浓度 与催化剂中毒程度之间的数学关系.

失活催化剂已被证明可采用不同的清洁剂清洗 再生, 且 Khodayari 等 ${ }^{[13]}$ 研究发现稀 $\mathrm{H}_{2} \mathrm{SO}_{4}$ 清洗比去 离子水具有更优的效果, 但是其并不能有效去除 $\mathrm{As}$ 元素. Peng 等 ${ }^{14}$ 发现采用浓度为 $4 \%$ 的 $\mathrm{H}_{2} \mathrm{O}_{2}$ 溶液清 洗可以除去部分 $\mathrm{AsO}_{x}$, 但同样会除去催化剂表面部 分 $\mathrm{V}_{2} \mathrm{O}_{5}$ 和沉积的硫酸盐, 而依然有大量 $\mathrm{AsO}_{2}$ 残留在 催化剂表面. $\mathrm{Li}$ 等 ${ }^{15}$ 提出浓度为 $4 \%$ 的 $\mathrm{Ca}\left(\mathrm{NO}_{3}\right)_{2}$ 可 以去除催化剂表面沉积的大部分 $\mathrm{AsO}_{x}$, 清洗后催化 剂脱硝活性恢复至 $80 \%$ 以上. 但两种清洁剂 $\mathrm{H}_{2} \mathrm{O}_{2}$ 和 $\mathrm{Ca}\left(\mathrm{NO}_{3}\right)_{2}$ 清洗后会导致催化剂表面活性组分的损失 严重. 因此, 研究开发高效 As 中毒催化剂清洗再生 方法具有十分重要的意义.

$\mathrm{FeCl}_{3}$ 溶液和重金属捕获剂 ( TMT) 溶液通常用 作清洗剂去除液体中的 As 化合物. 因此, 我们选择

收稿日期: 2020-06-12; 修回日期：2020-08-22.

基金项目: 江苏少重点研发计划(社会发展) 面上:项目(BE2017716) (Jiangsu Province Key R\&D Program (Social Development) General Project (BE2017716))

作者简介：赵重阴(1972-), 男, 高级下程师, 副总丁程师, 火电厂生产和科技研发等, Tel: 15810427971 , E-mail: chongyang.zhao@ chnenergy. com.cn (Zhao Chong-yang( 1972-), male, senior engineer, deputy chief engineer, thermal power plant production and technology research and development, etc., Tel: 15810427971, E-mail: chongyang.zhao@ chnenergy.com.cn).

* 通讯联系人, Tel:025-83790663, E-mail:amflora@ seu.edu.cn. 
其分别作为 As 中毒 SCR 催化剂再生清洗剂. 开展 As 中毒催化剂清洗再生研究, 调控清洗剂浓度及 清洗时间等因素，并结合通过 XRF，BET，SEM， XRD, XPS、 in situ IR 和 $\mathrm{H}_{2}$-TPR 等表征方法分析两 种再生剂清洗再生前后催化剂的化学组成、表面性 质和微观结构的变化情况, 获得 As 中毒催化剂清 洗再生方案.

\section{1 实验部分}

\section{1 材料与试剂}

SCR 脱硝催化剂活性测试台由南京工业大学搭 建. 烟气分析仪 (Testo350-XL) 购自德图仪器国际贸 易有限公司. 实验所用溶液纯度均为分析纯 $(A R)$, 其中, As 标准液纯度购自国药集团化学试剂有限 公司, $\mathrm{FeCl}_{3}$ 和 TMT 溶液购自阿拉丁试剂网; 催化 剂活性测试所用 $\mathrm{N}_{2} 、 \mathrm{NH}_{3} 、 \mathrm{NO} 、 \mathrm{O}_{2}$ 均由南京上元气 体有限公司提供.

\section{2 实验方法}

1.2.1 As 中毒催化剂制备实验所用新鲜蜂窝状 $\mathrm{V}_{2} \mathrm{O}_{5}-\mathrm{WO}_{3} / \mathrm{TiO}_{2} \mathrm{SCR}$ 催化剂购自中国大唐集团公 司，被切割成尺寸均为 $25 \mathrm{~mm} \times 25 \mathrm{~mm} \times 40 \mathrm{~mm}$ 的小 块, 其活性组分由 $1 \%$ (质量分数) $\mathrm{V}_{2} \mathrm{O}_{5}$ 和 $5 \%$ (质 量分数) $\mathrm{WO}_{3}$ 组成. 使用 $1000 \mathrm{ug} / \mathrm{mL}$ 的 As 标液, 以 $\mathrm{As} / \mathrm{V}$ 摩尔比为 $0.05 、 0.1 、 0.15 、 0.2$ 的比例将 4 块 催化剂浸渍在含有 As 标准溶液的烧杯中浸渍处理 $6 \mathrm{~h}$, 同时用磁力搅拌器尽可能实现均匀浸渍, 将催 化剂放人烘箱 $150{ }^{\circ} \mathrm{C}$ 下烘干, 在马弗炉中 $300{ }^{\circ} \mathrm{C}$ 下 焙烧 $6 \mathrm{~h}$, 制得模拟 As 中毒的催化剂, 记为 poisoned. 此外, 新鲜催化剂记为 fresh.

1.2.2 As 中毒催化剂再生 分别采用 $100 \mathrm{~mL}$ 去 离子水或不同浓度的再生溶液对 As 中毒催化剂进 行不同时间 (15、30、45 和 $60 \mathrm{~min}$ ) 超声清洗 ( 50 $\mathrm{kHz}, 300 \mathrm{~W}$ ) 再生. 同时, 通过 $\mathrm{NH}_{3} \cdot \mathrm{H}_{2} \mathrm{O}$ 将混合物 的 $\mathrm{pH}$ 值调节至 8 . 最后, 将清洗再生后的催化剂均 采用无水乙醇冲洗, 以去除催化剂表面残留的清洗 液, 为保持一致, 新鲜催化剂和 $\mathrm{As}$ 中毒催化剂同 样采用无水乙醇冲洗, 随后 $110{ }^{\circ} \mathrm{C}$ 下干燥 $5 \mathrm{~h}$, 然后 在连续气流 $(150 \mathrm{~mL} / \mathrm{min})$ 下在 $500{ }^{\circ} \mathrm{C}$ 下炦烧 $4 \mathrm{~h}$, 所获得的催化剂表示为 $x \mathrm{M}$, 其中 $x$ 代表不同的浓 度, M 代表不同的清洁溶液.

1.2 .3 催化剂活性测试方法催化剂活性测试在 固定床石英反应器中进行, 约 $100 \mathrm{mg}$ ( 0.450 0.280 $\mathrm{mm}$ ) 测试催化剂的活性. 进口气体由 $0.08 \%$ (体积
分数) $\mathrm{NO}+0.08 \%$ (体积分数) $\mathrm{NH}_{3}+5 \%$ (体积分数) $\mathrm{O}_{2}$ 组成, $\mathrm{N}_{2}$ 作为平衡气, 空速 $(\mathrm{SV})$ 为 $10000 \mathrm{~mL}$. $\mathrm{g}^{-1} \cdot \mathrm{h}^{-1}$. 采用气体分析仪 (Testo, 德国) 测量进出 口烟气中的 $\mathrm{NO}_{x}\left(\mathrm{NO}\right.$ 和 $\left.\mathrm{NO}_{2}\right)$ 浓度. 催化剂活性测 试的温度范围是 $200 \sim 500{ }^{\circ} \mathrm{C}$ ( 温度间隔 $50{ }^{\circ} \mathrm{C}$ ). 在 每个温度点, 保证测量温度稳定后, 进出口 $\mathrm{NO}_{x}$ 值 没有波动。

\section{$h=\left(\left[\mathrm{NO}_{x}\right]\right.$ in $\left.-\left[\mathrm{NO}_{x}\right]_{\text {oul }}\right) /\left[\mathrm{NO}_{x}\right]_{\text {in }} \times 100 \%$}

式中, $h$ 表示 $\mathrm{NO}_{x}$ 转化率, $\left[\mathrm{NO}_{x}\right]_{\text {in }}$ 和 $\left[\mathrm{NO}_{x}\right]_{\mathrm{out}}$ 分别为人口及出口处 $\mathrm{NO}_{x}$ 浓度.

As 去除率和活性组分 ( $V$ 和 $W$ ) 残余率分别采 用以下公式计算:

$w=($ As content of poisoned catalyst - As content of regenerated catalyst)/As content of poisoned catalyst $X$ $100 \%$

$r=V(W)$ content of regenerated catalyst/V (W ) content of poisoned catalyst $\times 100 \%$

1.2.4 催化剂表征 分别采用 ZSX Primus II 型 X 射线苂光光谱仪、V-Sorb2800P 型比表面积及孔径 分析仪、SmartLabTM X 射线衍射仪、Thermo Kalpha 高性能紧凑型 XPS 表面分析仪、Nicolet6700 型原位 漫反射傅立叶红外光谱仪和 FINSORB3010 型程序 升温化学吸附仪开展 SEM、BET、XRD、XPS、in situ IR 和 $\mathrm{H}_{2}$-TPR 等表征测试.

\section{2 结果与讨论}

\section{1 催化剂活性测试}

图 1 显示了用不同浓度的 $\mathrm{FeCl}_{3}$ 和 TMT 溶液对 As 中毒催化剂 SCR 活性清洗再生的影响. 从图 1 (a) 可以发现，相比于 As 中毒催化剂，不同浓度的 $\mathrm{FeCl}_{3}$ 溶液再生催化剂的活性均得到了明显的恢复, 其中, $20 \mathrm{mg} \cdot \mathrm{mL}^{-1} \mathrm{FeCl}_{3}$ 溶液再生催化剂在 $400{ }^{\circ} \mathrm{C}$ 下的 $\mathrm{NO}$ 转化率为 $90.57 \%$. 如图 1 (b) 所示, TMT 溶液清洗显示出对 As 中毒催化剂相似的再生效果. 随着 TMT 溶液浓度的增加, 再生催化剂的 $\mathrm{NO}_{x}$ 转化 率先上升后下降. $0.5 \% \mathrm{TMT}(\mathrm{pH}=8)$ 表现出最好的 再生效果, 特别是在 $400{ }^{\circ} \mathrm{C}$ 下 $\mathrm{NO}_{r}$ 转化率可以恢复 到 $96.50 \%$.

针对 $20 \mathrm{mg} \cdot \mathrm{mL}^{-1} \mathrm{FeCl}_{3}$ 和 $0.5 \% \mathrm{TMT}$ 清洗再生 情况, 分别研究了不同超声波清洗时间 (15、30、 45、60 min) 对 As 中毒催化剂再生效果的影响, 结 果如图 2 所示. 可以发现, 随着超声清洗时间从 15 增加到 $30 \mathrm{~min}, \mathrm{NO}_{r}$ 转化率增加, 而随着超声 

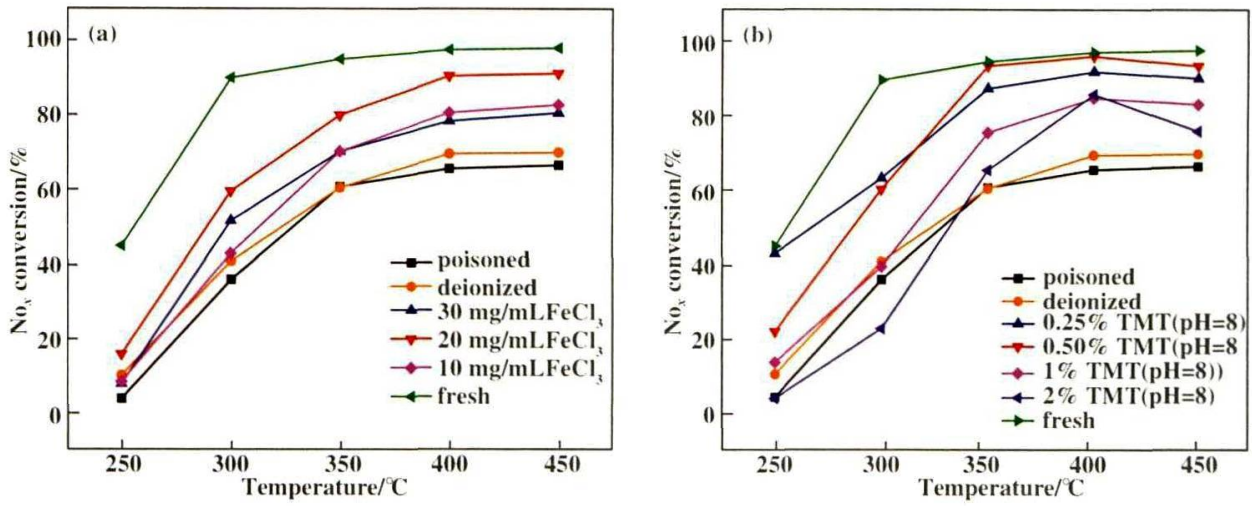

图 1 新鲜、中毒和清洗捅尘:催化剂 NO, 输化率

Fig. $1 \mathrm{NO}$, conversions of fresh, poisoned and cleaned regeneration catalysts
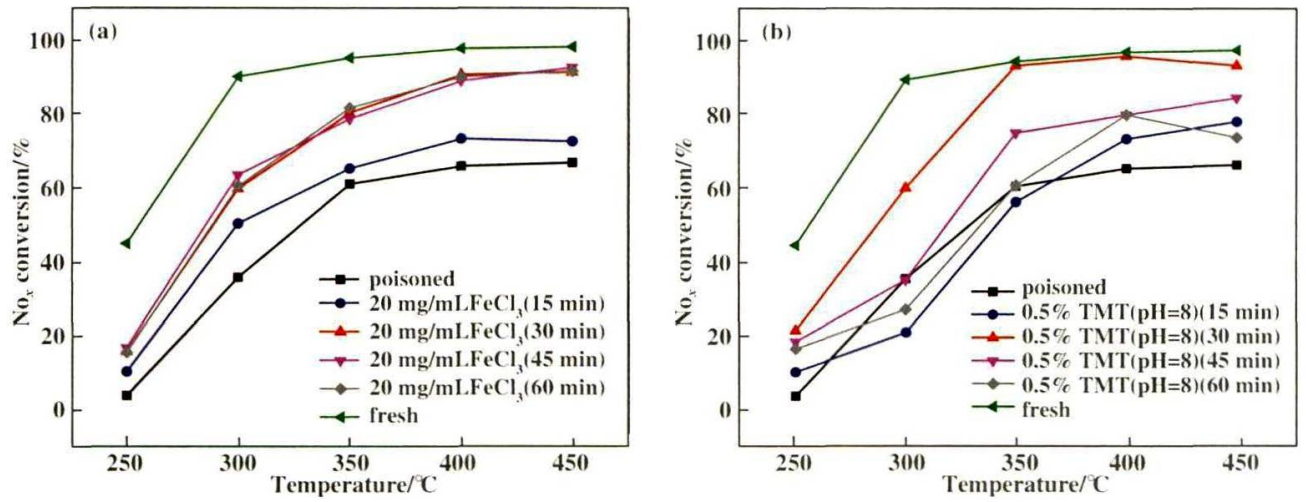

图 2 (a) $\mathrm{FeCl}_{3}$ (b) TMT 不同时间肪生: 催化剂的 NO, 转化率

Fig.2 NO conversions of regenerated catalysts with different times by (a) $\mathrm{FeCl}_{3}(\mathrm{~b}) \mathrm{TMT}$

清洗时间的进一步延长, $\mathrm{NO}_{\mathrm{x}}$ 转化率却略有降低. 当超声振荡清洗时间为 $30 \mathrm{~min}$ 时, 在 $350 \sim 400{ }^{\circ} \mathrm{C}$ 的温度范围内, 由 $20 \mathrm{mg} \cdot \mathrm{mL}^{-1} \mathrm{FeCl}_{3}$ 再生的催化剂

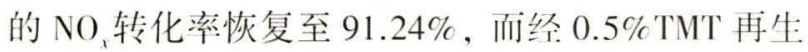

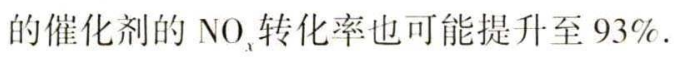

\section{$2.2 \mathrm{XRF}$ 分析}

采井 XRF 研究了用 $\mathrm{FeCl}_{3}$ 和 $0.5 \%$ TMT 溶液 (超卢振荡 $30 \mathrm{~min}$ ) 清洗的中毒催化剂中 As 的去除 率 $(83.67 \%$ 利 $94.57 \%$ ), 结果如表 1 所示. 去离子水 (deionized water) 清洗后仅能去除 $41.83 \%$ 的As, 而

表 1 不同溶液清洗 (30 min) 对 As 的去除率及活性组分残留比

Table 1 As removal and active components residual ratios by different solutions ( 30 min)

\begin{tabular}{|c|c|c|c|c|c|c|c|c|}
\hline & $\begin{array}{c}\text { Deionized } \\
\text { water }\end{array}$ & $\begin{array}{c}\mathrm{l} 0 /\left(\mathrm{mg} \cdot \mathrm{mL}^{-1}\right) \\
\mathrm{FeCl}_{3}\end{array}$ & $\begin{array}{c}20 /\left(\mathrm{mg} \cdot \mathrm{mL}^{-1}\right) \\
\mathrm{FeCl}_{3}\end{array}$ & $\begin{array}{c}30 /\left(\mathrm{mg}^{2} \cdot \mathrm{mL}^{-1}\right) \\
\mathrm{FeCl}_{3}\end{array}$ & $\begin{array}{c}0.25 \% \\
\text { TMT }\end{array}$ & $\begin{array}{l}0.5 \% \\
\text { TMT }\end{array}$ & $\begin{array}{l}1 \% \\
\text { TMT }\end{array}$ & $\begin{array}{l}2 \% \\
\text { TMT }\end{array}$ \\
\hline As removal rate $/ \%$ & 41.83 & 65.81 & 87.08 & 83.67 & 90.45 & 94.57 & 92.77 & 92.45 \\
\hline$V$ residual rate $/ \%$ & 99.81 & 97.14 & 95.16 & 90.32 & 96.75 & 98.15 & 94.62 & 91.00 \\
\hline W residual rate/\% & 98.89 & 96.19 & 96.26 & 97.14 & 98.74 & 98.47 & 98.53 & 98.46 \\
\hline
\end{tabular}

$20 \mathrm{mg} \cdot \mathrm{mL}^{-1} \mathrm{FeCl}_{3}$ 和 $0.5 \%$ TMT 表现阵交高的 As 去 除率, 分别为 $87.08 \%$ 和 $92.63 \%$. 其中, 再生催化剂 中 As 的残留量决定了其再生后的 $\mathrm{NO}$ ，催化脱除性 能. 值得注意的是, 较高的 As 去除率通常伴随着较 高的活性成分 (如 $\mathrm{V}_{2} \mathrm{O}_{5}$ 和 $\mathrm{WO}_{3}$ ) 损失. 如表 1 所示,
随着斗生溶液浓度的增加, $\mathrm{V}_{2} \mathrm{O}_{5}$ 的残留率呈现出轻 微的下降趋势, 而对另一活性组分 $W^{2} \mathrm{O}_{3}$ 的影响不明 显. 活性成分 $\mathrm{WO}_{3}$ 不受 $20 \mathrm{mg} \cdot \mathrm{mL}^{-1} \mathrm{FeCl}_{3}$ 的影响, 再生后 $\mathrm{WO}_{3}$ 的损失率不超过 $3 \%$. 在不同浓度下, 通过 TMT 再生的催化剂中, As 的去除摔相对较高, 
超过 90\%. TMT 的处理并不会导致活性组分 $\mathrm{WO}_{3}$ 的 严重损失，其保留率至少为 $98.47 \%$, 而活性组分 $\mathrm{V}_{2} \mathrm{O}_{5}$ 的损失随着 TMT 浓度的增加而增加.

\subsection{BET 分析}

新鲜、中毒和再生催化剂的结构性质测试结果 列于表 2 中. 新鲜 $\mathrm{V}_{2} \mathrm{O}_{5}-\mathrm{WO}_{3} / \mathrm{TiO}_{2}$ 催化剂的 BET 表

表 2 新鲜和清洗再生催化剂的织构性质

Table 2 Textural properties of the fresh and regenerated catalysts

\begin{tabular}{cccc}
\hline $\begin{array}{c}\text { Catalyst } \\
\text { samples }\end{array}$ & $\begin{array}{c}\text { BET surface } \\
\text { area } \\
\left(\mathrm{m}^{2} \cdot \mathrm{g}^{-1}\right)\end{array}$ & $\begin{array}{c}\text { Total pore } \\
\text { volume } \\
\left(\mathrm{cm}^{3} \cdot \mathrm{g}^{-1}\right)\end{array}$ & $\begin{array}{c}\text { Average pore } \\
\text { diameter } \\
/ \mathrm{nm}\end{array}$ \\
\hline fresh & 60.86 & 0.33 & 24.75 \\
poisoned & 49.28 & 0.32 & 26.27 \\
$20 \mathrm{mg} \cdot \mathrm{mL}^{-1} \mathrm{FeCl}_{3}$ & 64.11 & 0.35 & 21.89 \\
$0.5 \% \mathrm{TMT}^{\mathrm{T}}$ & 55.52 & 0.30 & 23.46 \\
\hline
\end{tabular}

面积为 $60.86 \mathrm{~m}^{2} \cdot \mathrm{g}^{-1}$, 而 $\mathrm{As}$ 中毒催化剂的比表面 积下降至 $49.28 \mathrm{~m}^{2} \cdot \mathrm{g}^{-1}$. 且相比于 fresh 催化剂 $(24.75 \mathrm{~nm})$, As 中毒催化剂平均孔径增加至 $26.27 \mathrm{~nm}$. 这是由于沉积的 $\mathrm{AsO}_{x}$ 阻塞了 $\mathrm{V}_{2} \mathrm{O}_{5}-\mathrm{WO}_{3}$ / $\mathrm{TiO}_{2}$ 催化剂表面的微孔和中孔. 但是, 以 $20 \mathrm{mg}$. $\mathrm{mL}^{-1} \mathrm{FeCl}_{3}$ 和 $0.5 \%$ TMT 进行再生可提高 BET 表面积 并降低催化剂的平均孔径, 这表明 $20 \mathrm{mg} \cdot \mathrm{mL}^{-1}$ $\mathrm{FeCl}_{3}$ 或 $0.5 \% \mathrm{TMT}$ 溶液清洗后, 沉积在催化剂表面 的大部分 $\mathrm{AsO}_{x}$ 被去除, 平均孔径减小, 气体接触通 道增加. 同时, 使得催化剂表面的活性位点重新暴 露出来, 进而参与 SCR 反应气体的吸附与活化.

\subsection{SEM 分析}

如图 3 所示, 在 As 中毒催化剂的表面上可以 观察到明显的网聚和沉积. 清洗再生后, 催化剂的 孔结构有一定程度恢复, 表面团聚明显减少. 催化 剂微孔结构的改善有利于气体的吸附.

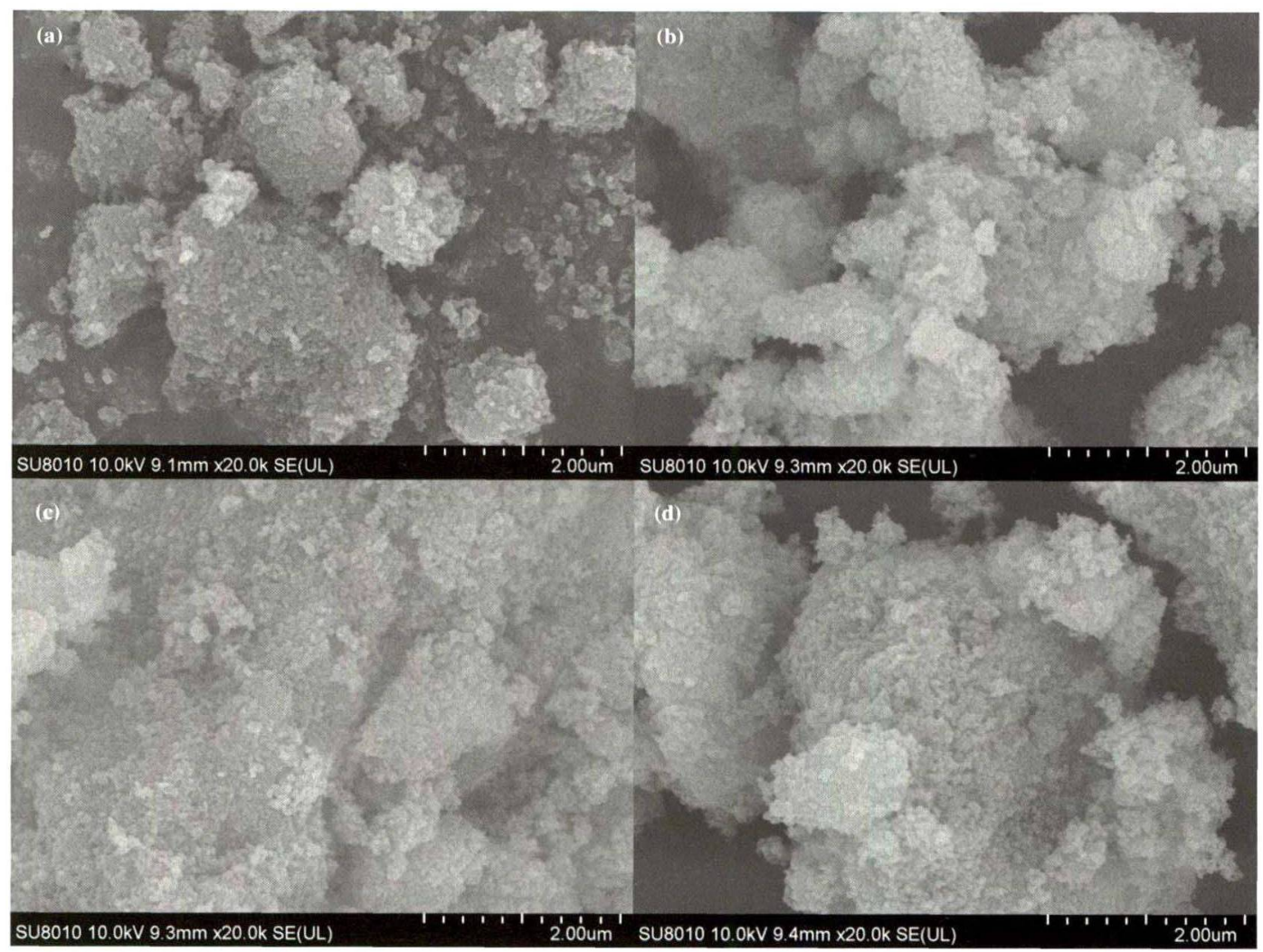

图 3 不同催化剂 SEM 图

Fig. 3 SEM images of different catalysts

(a) fresh; (b) regenerated by $20 \mathrm{mg} \cdot \mathrm{mL}^{-1} \mathrm{FeCl}_{3}$; (c) regenerated by $0.5 \%$ TMT; (d) poisoned

\subsection{XRD 分析}

新鲜、中毒和再生催化剂的 XRD 测试结果如 图 4 所示. 所有催化剂表面上均仅能检测到对应于
$\mathrm{TiO}_{2}$ (锐钛矿) 的衍射峰. 可以发现, As 中毒和 $20 \mathrm{mg} \cdot \mathrm{mL}^{-1} \mathrm{FeCl}_{3}$ 或 $0.5 \% \mathrm{TMT}$ 溶液再生后, 催化 剂的晶格结构既不受 As 中毒的影响, 也不受清洗 
液 $\mathrm{FeCl}_{3}$ 和 TMT 的影响 ${ }^{[16]}$.

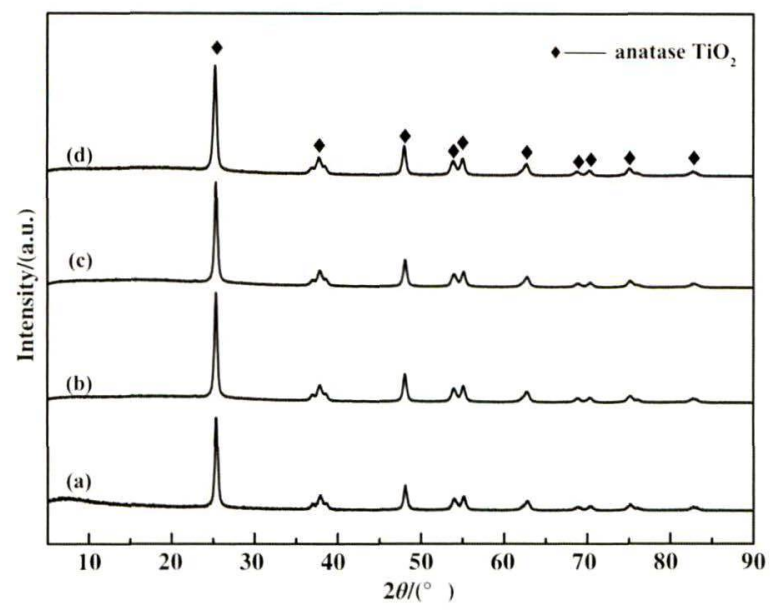

图 4 不同催化剂 XRD 图

Fig.4 XRD patterns of different catalysts

(a) poisoned; (b) regenerated by $20 \mathrm{mg} \cdot \mathrm{mL}^{-1} \mathrm{FeCl}_{3}$

$(30 \mathrm{~min})$; (c) regenerated by $0.5 \%$ TMT $(30 \mathrm{~min})$; (d) fresh

\subsection{XPS 分析}

图 5 为 As 中毒和清洗再生催化剂的 XPS 谱图. 根据已有研究, 结合能 $44.2 \sim 45.4 \mathrm{eV}$ 对应的是 $\mathrm{As}^{3+}$ $\left(\mathrm{As}_{2} \mathrm{O}_{3}\right), 45.7 \sim 47.8 \mathrm{eV}$ 对应的是 $\mathrm{As}^{5+}\left(\mathrm{As}_{2} \mathrm{O}_{5}\right)^{[17]}$. 可以发现, $20 \mathrm{mg} \cdot \mathrm{mL}^{-1} \mathrm{FeCl}_{3}$ 或 $0.5 \%$ TMT 溶液清 洗均有利于催化剂表面 $\mathrm{As}$ 的去除, 且 $\mathrm{As}_{2} \mathrm{O}_{3}$ 与 $\mathrm{As}_{2} \mathrm{O}_{5}$ 均可被清洗去除, 但是, 一部分 $\mathrm{AsO}_{x}$ 物种仍 残留于再生后的催化剂表面.

\subsection{In situ IR 分析}

作为 SCR 反应还原剂, $\mathrm{NH}_{3}$ 在催化剂的表面上 的吸附量以及吸附形态在 $\mathrm{NH}_{3}$ - SCR 脱硝反应是至 关重要的. 通过 $\mathrm{NH}_{3}$ 原位红外吸附实验对再生前后 催化剂表面酸位点的变化进行研究, 结果如图 6 所 示. 对于 fresh 催化剂 (图 6d), 在 1210 和 $1600 \mathrm{~cm}^{-1}$ 处的谱带分别与吸附在 Lewis 酸位点上的 $\mathrm{NH}_{3}$ 的对 称和不对称振动有关 ${ }^{18-19]} ; 1450$ 和 $1680 \mathrm{~cm}^{-1}$ 处的
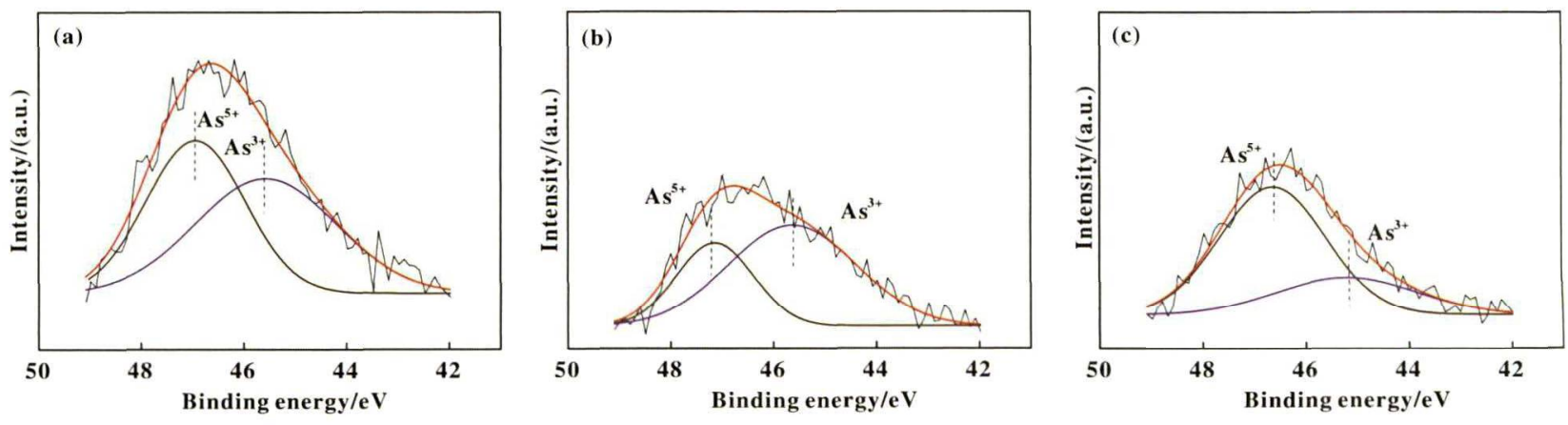

图 5 不同催化剂 As $3 d$ XPS 图

Fig. 5 As $3 d$ XPS patterns of different catalysts

(a) poisoned; (b) regenerated by $20 \mathrm{mg} \cdot \mathrm{mL}^{-1} \mathrm{FeCl}_{3}(30 \mathrm{~min}$ ); (c) regenerated by $0.5 \%$ TMT (30 min)

谱带由分别吸附于 Brønsted 酸位点上的 $\mathrm{NH}_{3}$ 的不对 称和对称振动引起 ${ }^{[20]}$.

对于 As 中毒催化剂 (图 6a), 1210 和 1600 $\mathrm{cm}^{-1}$ 处对应于 Lewis 酸位点上的 $\mathrm{NH}_{3}$ 的谱带消失 了, 而 1450 和 $1680 \mathrm{~cm}^{-1}$ 处对应于 Brønsted 酸位点 上的 $\mathrm{NH}_{3}$ 的谱带强度明显降低. 结果表明, $\mathrm{As}$ 的存 在严重影响了催化剂表面酸性位点的数量和强度, As 中毒催化剂表面酸位点数量明显减少, 尤其是 Lewis 酸位点.

从图 6(b 和 $\mathrm{c}$ ) 可以发现, $20 \mathrm{mg} \cdot \mathrm{mL}^{-1} \mathrm{FeCl}_{3}$ 或 $0.5 \%$ TMT 溶液清洗均有利于催化剂表面酸位点的 恢复. Lewis 酸位点的数量和强度在清洗再生后显 著增强, 这取决于在 $1600 \mathrm{~cm}^{-1}$ (Lewis 酸位点) 的带
强度的恢复.

\section{$2.8 \mathrm{H}_{2}$-TPR 分析}

图 7 显示了新鲜、As 中毒以及再生催化剂的 TPR 曲线. 根据图 7, 在 As 中毒催化剂上可以观察 到 3 个峰( 分别标记为峰 I, II 和 III) : $413^{\circ} \mathrm{C}$ 的峰 I 归因于 $\mathrm{V}^{5+} \rightarrow \mathrm{V}^{4+}$, 在 $469{ }^{\circ} \mathrm{C}$ 处的峰 II 分配至 $\mathrm{W}^{6+} \rightarrow$ $\mathrm{W}^{4+}$, 并且在 $600{ }^{\circ} \mathrm{C}$ 处的峰 III 分配为 $\mathrm{V}^{4+} \rightarrow \mathrm{V}^{3+}{ }^{15]}$. 可以发现, 用 $20 \mathrm{mg} \cdot \mathrm{mL}^{-1} \mathrm{FeCl}_{3}$ 溶液再生后, 催化 剂还原峰面积增加, 而还原峰却向较高温度段移 动. 而 $0.5 \%$ TMT 溶液清洗再生处理后, 催化剂峰面 积有所增加, 且还原峰向较低温度段移动. 这表明 $0.5 \%$ TMT 溶液清洗再生后催化剂的氧化能力恢复 优于 $20 \mathrm{mg} \cdot \mathrm{mL}^{-1} \mathrm{FeCl}_{3}$ 再生的催化剂. 

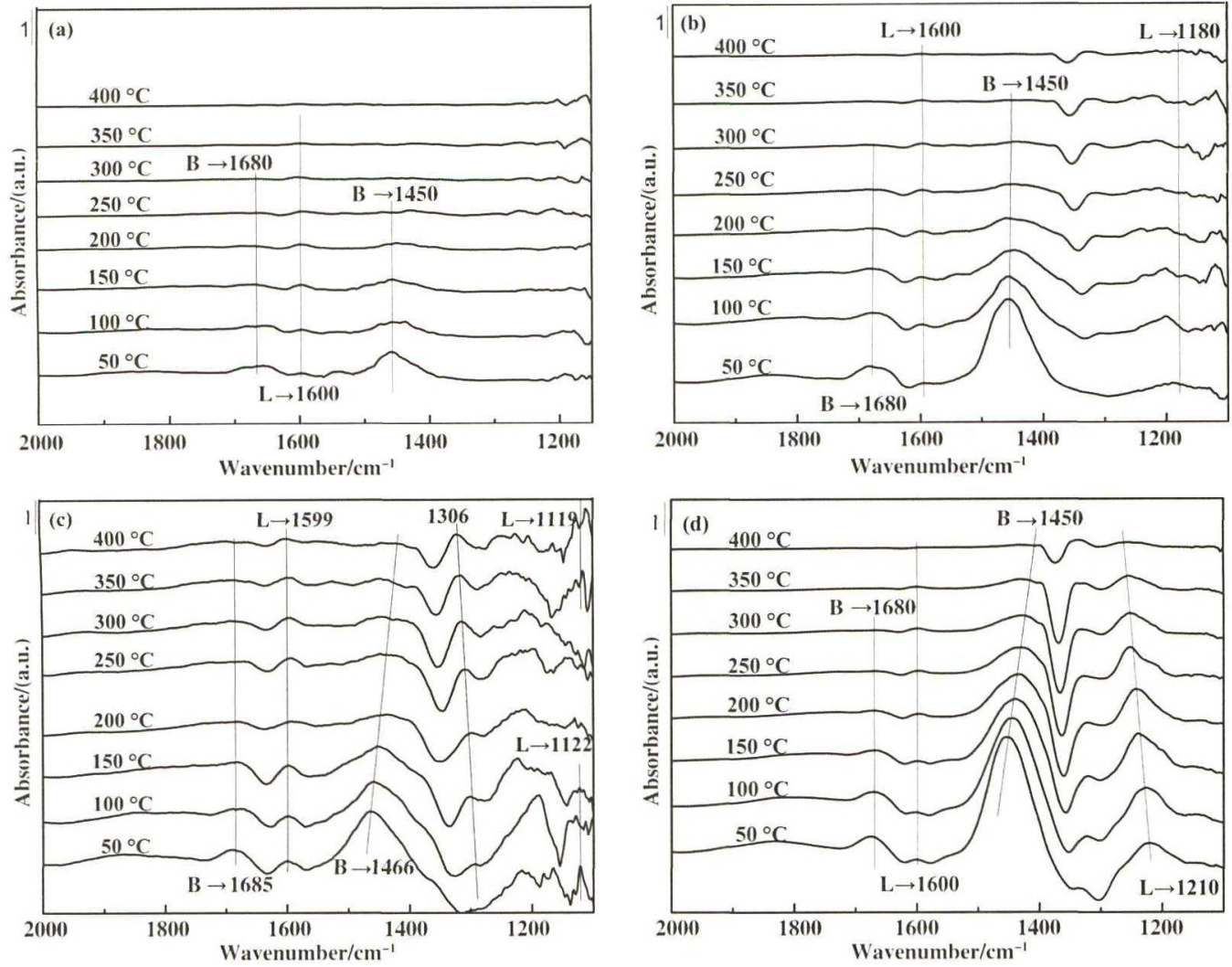

图6 不同催化剂 In situ DRIFT 图谱

Fig.6 In situ IR spectra of different catalysts

(a) poisoned; (b) regenerated by $20 \mathrm{mg} \cdot \mathrm{mL}^{-1} \mathrm{FeCl}_{3}(30 \mathrm{~min}$ ); (c) regenerated by $0.5 \%$ TMT (30 min) ; (d) fresh

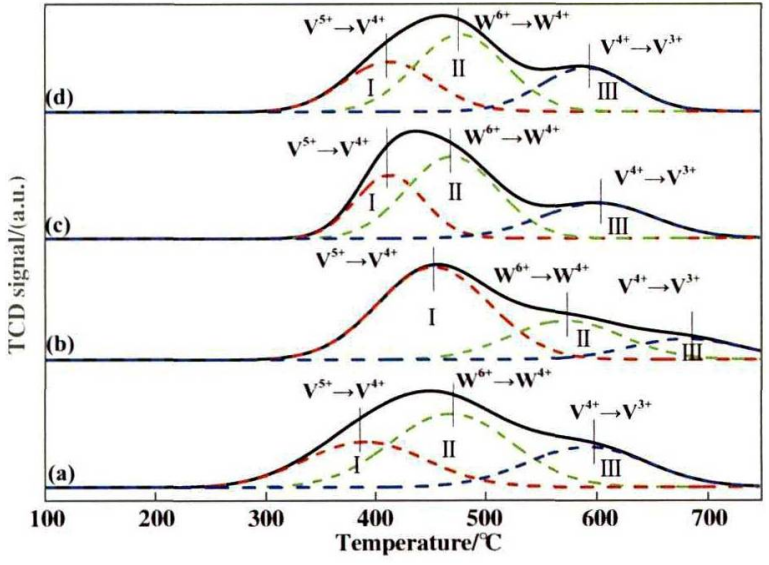

图 7 不同催化剂 $\mathrm{H}_{2}$-TPR 图

Fig. $7 \mathrm{H}_{2}$-TPR profiles of catalysts

(a) fresh; (b) regenerated with $0.5 \%$ TMT ( $30 \mathrm{~min})$;

(c) regenerated with $20 \mathrm{mg} \cdot \mathrm{mL}^{-1} \mathrm{FeCl}_{3}(30 \mathrm{~min})$ and (d) poisoned

$\mathrm{AsO}_{x}$ 沉积在催化剂表面, 致使催化剂表面活性 位点被覆盖, 而采用清洗液对中毒催化剂进行清洗
再生可去除部分 $\mathrm{AsO}$, 使活性位点重新暴露, 催化 剂活性有一定程度恢复. 但是, 部分 $\mathrm{AsO}_{x}$ 与催化剂 发生作用, 其与催化剂组分紧密结合, 清洗液难以 去除，对催化剂活性造成不可逆的损害. 同时，清 洗同样会导致部分活性组分损失，使得再生催化剂 的活性难以恢复至 100\% 。

\section{3 结论}

对于 $\mathrm{As}$ 中毒工业 $\mathrm{V}_{2} \mathrm{O}_{5}-\mathrm{WO}_{3} / \mathrm{TiO}_{2}$ 催化剂, 20 $\mathrm{mg} \cdot \mathrm{mL}^{-1} \mathrm{FeCl}_{3}$ 或 $0.5 \% \mathrm{TMT}$ 溶液清洗再生 $30 \mathrm{~min}$, 对催化剂表面 As 去除率分别为 $83.67 \%$ 和 $94.57 \%$. 当采用 $20 \mathrm{mg} \cdot \mathrm{mL}^{-1} \mathrm{FeCl}_{3}$ 溶液清洗再生时, 仅有部 分活性组分 $\mathrm{V}_{2} \mathrm{O}_{5}$ 的被洗去, 而使用 TMT 溶液时, $\mathrm{V}_{2} \mathrm{O}_{5}$ 和 $\mathrm{WO}_{3}$ 均被部分去除. 此外, 两种再生液清洗 再生后的催化剂均显示出良好的 SCR 活性, 且在 $400{ }^{\circ} \mathrm{C}$ 下采用 $20 \mathrm{mg} \cdot \mathrm{mL}^{-1} \mathrm{FeCl}_{3}$ 或 $0.5 \% \mathrm{TMT}$ 分别 清洗再生 $30 \mathrm{~min}, \mathrm{NO}$ 转化率分别从 $69.83 \%$ 恢复至 $90.57 \%$ 和 $96.50 \% . \mathrm{FeCl}_{3}$ 和 TMT 溶液均可优化中毒 催化剂的孔结构, 去除沉积在催化剂表面的 $\mathrm{AsO}_{x}$, 
增加比表面积和孔体积. As 的存在:降低了酸位点的 数量和强度, 特别是 Lewis 酸位点, 面 $\mathrm{FeCl}_{3}$ 或 TMT 溶液清洗再生后显著增加了催化剂表面的 Lewis 和 Brønsted 酸位点的数量和强度. 再生后中毒催化剂 的氧化能力得到了恢复, 尤其是采用 $0.5 \%$ TMT 清 洗 $30 \mathrm{~min}$ 后再生得到的催化剂.

\section{参考文献:}

[1] Casagrande L, Lietti L, Nova I, et al. SCR of NO by $\mathrm{NH}_{3}$, over TiO $\mathrm{T}_{2}$-supported $\mathrm{V}_{2} \mathrm{O}_{5}-\mathrm{MoO}_{3}$, catalysts: Reactivity and redox behavior [J]. Appl Catal B: Environ, 1999, 22( 1 ) : 63-77.

[2] Busca G, Lietti L, Ramis G, et al. Chemical and mechanistic aspects of the selective catalytic reduction of $\mathrm{NO}_{n}$, by ammonia over oxide catalysts: A review [J]. Appl Catal B: Enitron, 1998, 18(1/2): 1-36.

[3] Liu Z M, Woo S I. Recent advances in catalytic de $\mathrm{NO}_{4}$ science and technology [ J ]. Catal Rer, 2006, 48(1): $43-89$.

[4] Zhang Ya-ping (张亚平), Teng Yu-ling(滕玉婷), Lu Bing( 陠 斌), et al. Regeneration treatment technology of deactivated vanadium tungsten and titanium denitration (atalysts (夾活钒铇铁脱硝催化剂的青生处理技术) [J]. J Chinese Ceram Soc (硅酸盐学:报)，2019, 47 (4) : 440-449.

[5] Zhou Jin-hui(周锦晖), Li Guo-bo(李国波), Wu Peng (吴鹏), et al. The As poisoning mechanism over commercial $\mathrm{V}_{2} \mathrm{O}_{5}-\mathrm{WO}_{3} / \mathrm{TiO}_{2}$ catalyst ( 商业. $\mathrm{V}_{2} \mathrm{O}_{5}-\mathrm{WO}_{3} / \mathrm{TiO}_{2}$ 脱硝催化剂砷中毒机理) [J]. J Mol Catal (China)(分 子催化), 2018, 32(5): 55-64.

[6] Shen Bo-xiong( 沈伯雄), Xiong Li-xian(熊网仙l), Liu Ting(刘亭). Study on arsenic poisoning of supported $\mathrm{V}_{2} \mathrm{O}_{5}-\mathrm{WO}_{3} / \mathrm{TiO}_{2}$ catalyst ( 负载型 $\mathrm{V}_{2} \mathrm{O}_{5}-\mathrm{WO}_{3} / \mathrm{TiO}_{2}$ 催化 剂的砷中1毒研究) [J]. J Fuel Chem Technol ( 燃料化学: 学:报), 2011, 39(11): 856-859.

[7] Guo X, Zheng C G, Liu Y H, et al. The study on the mode of occurrence of mercury, arsenic and selenium in coal [ J]. J Eng Thermo, 2001, 22(6): 763-766.

[8] Zheng L. G, Liu G J, Chou C L, et al. Arsenic in Chinese coal: Its abundance, distribution, moles of occurrence, enrichment processes, and environmental significance [J]. Acta Geoscientica Sinica, 2006, 27(4) : 355-366.

[9] Peng Y, Li J H, Si W Z, et al. Insight into deactivation of commercial SCR catalyst by arsenic: An experiment and DFT study $[\mathrm{J}]$. Environ Sici Technol, 2014, 48 (2.3) : 13895-13900.

[10] Kong M, Liu Q C. Wang X Q, et al. Performance impact and poisoning mechanism of arsenic over commercial $\mathrm{V}_{2} \mathrm{O}_{5}-\mathrm{WO}_{3} / \mathrm{TiO}_{2}$, SCR catalyst $[\mathrm{J}]$. Catal Commun, $2015,72(5): 121-126$.

(11] Jian D, Liu Q C, Ming K, et al. Influence of arsenic in flue gas on the performance of $\mathrm{V}_{2} \mathrm{O}_{5}-\mathrm{IOO}_{3} / \mathrm{TiO}_{2}$ catalyst in selective catalytic reduction of $\mathrm{NO}, \ldots, \mathrm{J}$. Journal of $\mathrm{Fu}$ el Chemistry \& Technology, 2016, 44(4) : 495-499.

[12] Sun K Q, Zhong Q, Yu A H. Study on arsenic poisoning of SCR catalyst $[\mathrm{J}]$. China Environmental Protection Industry, 2008, 2008( 1): 40-42.

[13] Khodayari R, Odenbrand C U I. Regeneration of commer(rial Ti(O) $-\mathrm{V}_{2} \mathrm{O}_{5}-\mathrm{WO}_{3}$, SCR catalysts used in bio fuel plants J . Appl Catal B: Emviron, 2001, 30(1/2) : 8799 .

14] Peng Y, Li J H, Si W Z, et al. Deactivation and regeneration of a commercial SCR catalyst: Comparison with alkali metals and arsenic $[\mathrm{J}]$. Appl Catal B: Environ, 2015, 168/169: 195-202.

[15] Li X, L.i J H, Peng Y, et al. Regeneration of commercial SCR catalysts: Probing the existing forms of arsenic oxide [J]. Eniron Sci Technol, 2015, 49(16) : 9971-9978.

[16] Ramos L. M A, Cedeño C L. Effect of sulfates and reduced-vanadium species on oxidative desulfurization (ODS) with $\mathrm{V}_{2} \mathrm{O}_{5} / \mathrm{TiO}_{2}$ catalysts [ J]. Ind Eng Chem Res, 2010, 50(5) : 2641-2649.

[17] Fu B, Hower J C, Dai S F, et al. Determination of chemical speciation of arsenic and selenium in High-As coal combustion Ash by X-ray photoelectron spectroscopy: Examples from a kentucky stoker Ash [J]. ACS Omega, 2018, 3(12): 17637-17645.

18] Zhang Y P, Wang X L, Shen K, et al. W(O) modification of $\mathrm{MnO} / \mathrm{TiO}_{2}$ catalysts for low temperature selective catalytic reduction of $\mathrm{NO}$ with ammonia [J]. Chin J Catal, 2012, 33(9/10): 1523-1531.

[19] Pan S W, Luo H C, Li L, et al. $\mathrm{H}_{2} \mathrm{O}$ and $\mathrm{SO}_{2}$, deactivation mechanism of $\mathrm{MnO}_{1} / \mathrm{MWCNT}$ s for low-temperature SCR of $\mathrm{NO}_{4}$, with $\mathrm{NH}_{3}[\mathrm{~J}]$. J Mol Catal A: Chem. 2013, 377: 154-161.

[20] Topsoe N Y, Topsoe H, Dumesic J A I. Combined temperature-programmed in-situ FTIR and on-line massspectroscopy studies [J]. J Catal, 1995, 151(1) : 226240. 


\title{
Study on Regeneration of Commercial $\mathrm{V}_{2} \mathrm{O}_{5}-\mathrm{WO}_{3} / \mathrm{TiO}_{2}$ Catalyst for Arsenic Poisoning
}

\author{
ZHAO Chong-yang ${ }^{1}$, LI Guo-bo ${ }^{2}$, SUI Hua-jun ${ }^{1}$, LIU Chu-ming ${ }^{1}$, WANG Ling $^{2}$, \\ ZHANG Ya-ping ${ }^{2 *}$ \\ (1. China Energy Jianbi Power Plant, Zhenjiang 212006, China;
}

2. School of Energy and Environment, Southeast University, Nanjing 210096, China)

\begin{abstract}
FeCl}_{3}$ and 2,4,6-trimercaptotriazine (TMT) solutions were used to clean and regenerate arsenic (As) poisoned commercial $\mathrm{V}_{2} \mathrm{O}_{5}-\mathrm{WO}_{3} / \mathrm{TiO}_{2}$ catalysts. A comparative study of physico-chemical properties between poisoned and regenerated catalysts was carried out by Brunauer-Emmette-Teller (BET), X-ray diffraction (XRD), $\mathrm{X}$-ray fluorescence $(\mathrm{XRF}$ ), in situ infrared spectrometry (in situ IR), and temperature programmed reduction of $\mathrm{H}_{2}\left(\mathrm{H}_{2}\right.$-TPR $)$. It was found that the catalyst's denitration activity was greatly restored after cleaning. Regeneration by $20 \mathrm{mg} \cdot \mathrm{mL}^{-1} \mathrm{FeCl}_{3}$ and $0.5 \%$ TMT solution for $30 \mathrm{~min}$ exhibited best As removal ratio of $83.67 \%$ and $94.57 \%$, respectively. The micropores and mesopores which blocked by As oxides were cleaned, thus the specific surface area, pore volume increased and the average pore diameter decreased. Meanwhile, the Brønsted and Lewis acid strengths of the catalyst surface increased after $\mathrm{FeCl}_{3}$ and 2,4,6-TMT solution cleaning and regeneration, which might be the main reason for the improvement of catalytic performance over the regenerated catalysts.
\end{abstract}

Key words: selective catalytic reduction; catalyst ; arsenic poisoning; regeneration 